\title{
EDUCACIÓN, TECNOLOGÍA Y SUBJETIVIDAD CONTEMPORÁNEA
} EDUCATION, TECHNOLOGY AND CONTEMPORARY SUBJECTIVITY

\author{
Dr. Carlos Adolfo Rengifo ${ }^{1}$ \\ Universidad de San Buenaventura
}

Este número de la Revista Boletín Redipe recoge en gran parte artículos de investigación y reflexión propositiva en torno a la tecnología y su aplicación en la educación y otras esferas de la vida productiva y social, y otros sobre temas inherentes a las áreas y campos de la educación. Inicia con un ensayo potente del pensador Fernando Cruz Kronfly que seguramente puede alentar la labor educativa consagrada a formar mejores seres humanos, individuos con una consciencia actuante capaz de resistir

1 Estudios Postdoctorales Università degli studi dell Insubria, Varese - Italia. Doctor en Filosofía de la Universidad Pontifica Bolivariana de Medellín. Magíster en Filosofía de la Universidad del Valle. Profesional en Filosofía de la Universidad del Quindío y Especialista en Pedagogía y docencia Universitaria de la Universidad La Gran Colombia Armenia. Investigador senior en Colciencias. Docente investigador de la Universidad San Buenaventura de Cali. Investigador de la Red Iberoamericana de Pedagogía, Redipe. Integrante de los grupos de investigación: Educación y Desarrollo Humano y Paideia. Editor in chief de la Revista Guillermo de Ockham. Correo director.maestriadesarrollohum@ usbcali.edu.co, careca1106@gmail.com. orcid.org/00000001-5737-911X al instalamiento del "tipo de subjetividad" obrado por el orden financiero, político y económico dominante en los habitantes contemporáneos para dejarlos convertidos en almas gemelas, al compás rítmico de su dinámica deshumanizante en virtud de la cual la maximización del capital está por encima de la vida humana y planetaria.

\section{LA SUBJETIVIDAD CONTEMPORÁNEA: ALMA GEMELA DEL CAPITALISMO ACTUAL.} Ensayo de reflexión propositiva a cargo del pensador colombiano, académico y escritor, Fernando Cruz Kronfly, Universidad del Valle, en torno a un fenómeno de sujetación del individuo contemporáneo por parte del capitalismo. Hace aproximadamente 40 años, Frederik Jameson escribió, términos más, términos menos: "es más fácil imaginar el fin del mundo que el fin del capitalismo". Lo anterior, traído a valor presente, lleva a pensar que la garantía de "eternidad" , 
intangibilidad y reproducción del capitalismo proviene ahora del tipo de subjetividad que logró instalar en los habitantes contemporáneos, para dejarlos convertidos en almas gemelas suyas. Seres humanos mutilados de utopías de futuro, en determinadas coyunturas solo rabiosos manifestantes callejeros incapaces de luchar contra el modelo e imaginar su destrucción y su fin. Por el contrario, y a pesar de las miserias, procuran integrarse a sus favores y beneficios. De este fenómeno cultural y social inédito se ocupa el presente ensayo.

\section{ESTRATEGIAS PARA CONSTRUIR LA} PAZ EN LA REGIÓN. CONTRIBUCIONES DE LA FACULTAD DE EDUCACIÓN DE LA UNIVERSIDAD DE NARIÑO. Artículo de investigación autoría de los académicos Nelson Torres Vega, Francisco Javier Torres Martínez, Edgar Guillermo Mesa Manosalva, Grupo GIDEP de la Universidad de Nariño. Derivado del proyecto: Estrategias pedagógicas para construir la paz en la región: Contribuciones de la Facultad de Educación, Universidad de Nariño. Analiza las estrategias pedagógicas para construir la paz en la región, como una contribución de la Facultad de Educación. En el marco de los diálogos de paz se ha invitado a todas las universidades del país a generar sugerencias, a presentar propuestas, iniciativas y estrategias orientadas a construir una paz duradera que aporte al desarrollo económico, político, social y cultural. Entre otras conclusiones se destaca que la Facultad de Educación de la Universidad de Nariño, en su Proyecto Educativo, concibe el concepto de la didáctica, como las teorías y prácticas que en coherencia con la fundamentación pedagógica y en consideración a la naturaleza, la historia y la epistemología de los saberes, permiten desarrollar procesos de transposición didáctica que conllevan a la apropiación consciente y reflexiva del conocimiento científico. Lo anterior, constituyen insumos insustituibles para la construcción de estrategias de paz con miras a fortalecer los procesos derivados del acuerdo de la Habana y el post-acuerdo, la cultura y la educación para paz, el pacifismo, la resolución de conflictos, la mediación, entre otros, para contribuir a la construcción de la paz en el departamento de Nariño.

ECONOMÍA DEL CONOCIMIENTO EN LA EDUCACIÓN SUPERIOR: FACTOR CLAVE EN LA CALIDAD EDUCATIVA. Artículo de investigación autoría de los académicos Byron Medina Delgado, Wlamyr Palacios Alvarado, Luis Leonardo Camargo Ariza, UFPS_ Unimagdalena. Analiza algunos fundamentos teóricos y metodológicos con base en resultados de algunas investigaciones anteriores relacionadas con la calidad educativa a nivel superior y con la economía del conocimiento. Las instituciones de educación superior tienen directa incidencia en el desarrollo de los países a través de un proceso continuo que apunta hacia la construcción de conocimientos para generar beneficios, calidad de vida en la población y desarrollo humano de los habitantes; escenario en el que a su vez, los profesionales de las diversas disciplinas son determinantes cuando se han beneficiado de una educación superior de alta calidad, en donde la economía del conocimiento ejerce un papel preponderante. Señala que la evaluación de la calidad de los servicios educativos ofrecidos por la educación superior está relacionada con la acreditación ante el CNA.

PRÁCTICA PEDAGÓGICA Y EXPERIENCIA PROFESIONAL: FORTALEZAS $Y$ OPORTUNIDADES DE MEJORA. Artículo de investigación a cargo de los académicos

Yuretsy Alexandra Reyes Cáceres, Raúl Prada Núñez, Audin Aloiso Gamboa Suárez, Grupo de investigación de Investigación en Pedagogía y Práctica Pedagógica. Indaga sobre las fortalezas y oportunidades de mejora en las prácticas pedagógicas de maestros de matemáticas en 15 instituciones educativas de Norte de Santander. Los resultados permitieron identificar que 
todos los aspectos que envuelven la práctica pedagógica se correlacionan significativamente entre ellos. Se concluye que los docentes en sus primeros años de experiencia no realizan ningún estudio posgradual, sino que se dedican solo a trabajar y satisfacer sus necesidades familiares y económicas.

LA EDUCACIÓN AMBIENTAL: UNA TAREA INCONCLUSA DESDE LOS PROYECTOS AMBIENTALES ESCOLARES. Artículo de investigación de los académicos Nabi Del Socorro Pérez-Vásquez, Edith De Jesús Cadavid-Velásquez, Elvira Patricia FlórezNisperuza, Universidad de Córdoba. Caracteriza el estado actual de los PRAEs de las Instituciones Educativas (IE) oficiales urbanas y rurales de educación básica y media del municipio de Montería, en relación con los requerimientos normativos en su construcción y su dinamización. Los resultados evidencian que, en la mayoría de las IE, los proyectos se ejecutan de manera aislada a la gestión académica, dejando de lado principios didácticos de transversalidad, interdisciplinariedad e integralidad del currículo y generalmente liderados por los profesores de Ciencias Naturales. Se concluye que estos proyectos no han permitido consolidar una educación ambiental integral que tengan en cuenta los aspectos naturales y socioculturales y el mejoramiento de la calidad de las comunidades educativas.

\section{LOS SIMULADORES EDUCATIVOS COMO INSTRUMENTO PEDAGÓGICO PARA LA ENSEÑANZA DE LAS FINANZAS. Artículo} de investigación, autoría de los académicos Mawency Vergel Ortega, Luisa Stella Paz Montes, Diego Mauricio Álvarez Paz, Universidad. Visualiza las competencias asociadas al pensamiento financiero mediante el uso de herramientas tecnológicas, en especial de simuladores, en los estudiantes de educación superior. Entre los resultados se encontró que a través de la utilización de simuladores, los docentes pueden apropiarse de las TIC como mecanismo de enseñanza más asertivo para estimular el pensamiento financiero y económico en los estudiantes.

\section{LA INFLUENCIA DE LA MATEMÁTICA EN EL DESARROLLO DEL PENSAMIENTO. Artículo} de revisión elaborado por Oscar Alejandro Guaypatin Pico, Silvia Liliana Fauta Ramos, Xavier Alexander Gálvez Cisneros y Diana Montaluisa, Universidad Técnica de Cotopaxi. Indaga acerca de la importancia de las matemáticas para el desarrollo del pensamiento de los estudiantes, en virtud del cual sean seres lógicos, críticos, dispuestos a razonar. El pensamiento matemático conlleva actitudes, valores y capacidad de crear nuevas ideas, soluciones y preguntas novedosas. Todas las personas nacen con la capacidad de desarrollar la inteligencia, las diferentes capacidades dependerán de la estimulación que le demos a cada forma de pensamiento. Para lograr un desarrollo adecuado necesitamos usar métodos y técnicas desde muy temprana edad dado que las matemáticas son fundamentales en la vida cotidiana y en el ámbito estudiantil para poder entender, comprender y analizar conceptos abstractos y formular metas y logros personales. De esta manera se estará más cerca de atender problemas en diferentes ámbitos de la vida.

CARACTERIZANDO LA RESOLUCIÓN DE PROBLEMAS DESDE LA VARIACIÓN $Y$ EL CAMBIO EN DOMINIOS DISCRETOS $Y$ LA TEORÍA FUNDAMENTADA. Artículo de investigación autoría de los académicos Luis Fernando Mariño, Rosa Virginia Hernández, Universidad Francisco de Paula Santander, em torno a la pregunta: ¿Cómo es la naturaleza de la resolución de problemas que involucran ecuaciones lineales diofánticas de la forma , manifestado por profesores de matemáticas en formación? Entre los hallazgos se destacan las acciones variacionales manifestadas por los participantes, que les posibilitó ir de sustituciones 
y combinaciones variacionales a establecer relaciones para formalizar, generalizar, probar, organizar y reorganizar su conocimiento.

\begin{tabular}{lcr} 
APROPIACIÓN DE & \multicolumn{2}{c}{ COMPETENCIAS } \\
COMUNICATIVAS & Y TECNOLÓGICAS \\
EN MAESTROS EN FORMACIÓN DE UN \\
PROGRAMA ACREDITADO DE ALTA
\end{tabular}

CALIDAD. Artículo de investigación elaborado por los académicos Raúl Prada Núñez, Audin Aloiso Gamboa Suarez, William Rodrigo Avendaño Castro, GIPEPP. Dirigido a la identificación del nivel de apropiación de competencias comunicativas y tecnológicas que tienen estudiantes de un programa de Licenciatura en Matemáticas en una universidad pública en Norte de Santander. Los resultados evidencian la evolución del desarrollo de estas competencias a medida que el estudiante avanza en su proceso de formación curricular, lo cual es un elemento a favor de la propuesta curricular que posee el programa académico.

COMPONENTE TECNOLÓGICO: ARTICULACIÓN CON EL ÁREA EDUCACIÓN FÍSICA PARA PROMOVER LA FORMACIÓN INTEGRAL EN LA ESCUELA. Artículo de investigación científica y tecnológica elaborado por los académicos Nicol Pinilla Fonseca, María Cristina Gamboa Mora, Gerardo Patiño Varón, Universidad de Baja California. TepicMéxico. Dirigido a establecer la percepción sobre la formación integral de los estudiantes de una institución educativa de Tolima-Colombia, obtenida tras la implementación de estrategias inclusivas de tecnología y Tecnologías de la Información y la Comunicación, con procesos motrices y corporales. Se concluye que las estrategias empleadas tienen efecto sobre la formación integral, dado que las medias de percepción aumentaron significativamente.

INDICADORES TECNOLÓGICOS PARA LA SELECCIÓN DE PLATAFORMAS MOOC. Artículo de investigación autoría de las académicas Susan Yulieth Huertas Lòpez,
Fredy Yesid Mesa Jiménez, UPTC. Analiza los aspectos tecnológicos que intervienen en la calidad de un curso virtual. Para tal fin se realiza una revisión documental, encuestas, observaciones y exploraciones para establecer algunos indicadores que permitan la valoración de aspectos tecnológicos en plataformas MOOC, permitiendo establecer indicadores en tres tipologías: 1) tecnología de equipo, 2) tecnología de operación y 3) tecnología de producto, a través de los cuales se puede realizar una valoración cuantitativa y cualitativa de manera secuencial dentro del desarrollo de un curso, entonces si se quiere realizar un MOOC se debe establecer los componentes tecnológicos requeridos de acuerdo a la necesidad. La utilidad de las plataformas de Cursos Abiertos Masivos en Línea o MOOC (Massive Open Online Courses) permite desarrollar experiencias formativas personalizadas, con la ventaja de que estos cursos son ofrecidos a través de entornos virtuales, los cuales pueden ser accedidos a cualquier hora y desde cualquier parte del mundo, por medio del internet. Dada la alta oferta en educación electrónica, ¿Cómo se puede seleccionar un curso que satisfaga los criterios de calidad y confiabilidad?

\section{TECNOLOGÍAS DISRUPTIVAS EN EDUCACIÓN VIRTUAL. Artículo de} investigación obra de los académicos Yolanda González Castro, Omaira Manzano Duran, Marleny Torres Zamudio Universidad Nacional Abierta y a Distancia UNAD. Incluye una revisión documental de bases de datos científicas y en lo cuantitativo se realizó un diseño no experimental longitudinal de tipo descriptivo apoyado en las fases de la vigilancia tecnológica. Mediante el empleo de la base de datos de Scopus y el software VOSViewer se determinaron los siguientes clústeres a) características del e-learning, (b) características de la industria 4.0, (c) avances tecnológicos tradicionales para la educación y la industria y (d) avances tecnológicos disruptivas para la educación 
virtual y la industria. Entre las tecnologías que tienen mayor incidencia en el campo de la educación virtual están: el machine learning, la inteligencia artificial, la minería de datos, el internet de las cosas, la realidad virtual, realidad aumentada y sistemas embebidos. La cuarta revolución industrial está impactando el ámbito empresarial, por lo tanto, se hace necesario que las organizaciones educativas virtuales estén preparadas para innovar en sus procesos.

\section{USO DE SIMULADORES PHET PARA EL APRENDIZAJE DEL CONCEPTO DE SOLUCIONES DESDE LAS} REPRESENTACIONES EN QUÍMICA. Artículo de investigación de los académicos Andis Rafael Pacheco, Danny José Lorduy, Elvira Patricia Flórez, Julio César Páez, Grupo de Investigación en Ciencias Naturales y Educación Ambiental, GICNEA. Facultad de Educación y Ciencias Humanas. Universidad de Córdoba, Colombia. Analiza el uso de simuladores PhET asociados con actividades experimentales para el aprendizaje del concepto de soluciones desde las representaciones en química en estudiantes de grado décimo de una Institución Educativa pública del departamento de Córdoba, Colombia. Los resultados muestran que el uso de simuladores $\mathrm{PhET}$ asociados con actividades experimentales promovieron la construcción de aprendizajes mejor estructurados y más profundos en torno al concepto de soluciones químicas por parte de los estudiantes, mediante la movilidad mental entre la información abstracta e implícita y representaciones macro o explícitas. La investigación contribuyó significativamente a una nueva visión en las clases de química, brindando alternativas de aprendizaje de forma dinámica e interactiva con diferentes recursos que se pueden aplicar a todos los contenidos curriculares de un curso de química.

VALORACIÓN PATRIMONIAL A PARTIR DEL ANÁLISIS MORFOLÓGICO Y MATEMÁTICO DE SAN PEDRO Y SAN PABLO, DE LA
CATEDRAL DE SAN JOSE DE CÚCUTA. Artículo de investigación Julio Alfredo Delgado Rojas, Yannette Díaz Umaña, Mawency Vergel Ortega, Universidad Francisco de Paula Santander. Socializa los resultados de los análisis morfológicos y matemáticos de las figuras en mármol de San pedro y San Pablo, en donde se exponen sus orígenes artísticos, geográficos, y la descripción de los procesos artísticos a la que fueron sometidas estas dos grandes piezas de mármol, confirmándose la procedencia del material en el Monte altissimo de Pietrasanta a manos del escultor Pietro Bibolotti. Igualmente se determina el origen antropométrico en la Grecia clásica, con el canon estándar, por cuanto sus proporciones matemáticas. De ahí se reseña un alto valor artístico y patrimonial, de estas dos esculturas de la catedral de San José de Cúcuta.

\section{SELF-CONFRONTATION: A GATEWAY TOWARDS THE UNDERSTANDING OF ENGLISH TEACHERS' PEDAGOGICAL} PRACTICE. Artículo de investigación de la académica María Fernanda Téllez Téllez , Facultad de Ciencias de la Educación, Universidad Externado de Colombia. Muestra los avances preliminares de una investigación fenomenológica hermenéutica. El propósito del estudio fue develar qué conocimientos surgieron de la reflexión sobre la acción de las prácticas de los participantes a través de la autoconfrontación. Los instrumentos para la recolección de datos incluyeron una entrevista semiestructurada, un grupo focal, las trascripciones de video de las entrevistas de autoconfrontaciones y notas de campo. Se utilizó el análisis temático para interpretar los datos. Los hallazgos iniciales demostraron que M1 reflexionó sobre sus prácticas apoyándose en sus saberes empírico, experiencial y reflexivo. 
GAMIFICACIÓN Y EVALUACIÓN FORMATIVA EN LA ASIGNATURA DE MATEMÁTICA A TRAVÉS DE HERRAMIENTA WEB 2.0. Artículo de investigación obra de los académicos Raúl Prada Núñez, César Augusto Hernández Suárez, William Rodrigo Avendaño Castro, UFPS. Dirigido a comprender la gamificación, la evaluación formativa en una herramienta web2.0, y su mejora en la práctica educativa de las matemáticas en un contexto digital. La investigación se contextualizó dentro del enfoque cualitativo, el tipo fue investigaciónacción. Se halló que la evaluación formativa en una herramienta Web de la asignatura de matemática en un ambiente no lúdico y gamificado depende de los métodos de realimentación utilizados por el docente en dar respuestas a las inquietudes y solicitudes presentadas por los estudiantes. Se concluyó que cuanto más aumenta la gamificación en sus elementos y la evaluación formativa en el contexto digital más cambia significativamente el aprendizaje de las matemáticas.

\section{HIGHER EDUCATION IN THE FRAMEWORK} OF THE BEHAVIORAL ECONOMY OF GENERATION Z IN COLOMBIA. Artículo de investigación elaborado por los académicos Byron Medina Delgado, Wlamyr Palacios Alvarado, UFPS y Luis Leonardo Camargo Ariza, Universidad del Magdalena. Explora las teorías y conceptos sobre la economía del comportamiento y la generación Z, con el fin de relacionarlos con la educación superioren materia de identificación de las carreras universitarias de mayor preferencia de parte de esta generación. La economía del comportamiento estudia las dimensiones cognitivas, emocionales y sociales inherentes al obrar del ser humano; sus análisis e investigaciones recientes señalan su importancia en la toma de decisiones según variables del contexto y características personales del individuo como en el caso de la generación $Z$. Como conclusión general, es importante señalar que este enfoque permite sentar las bases para futuras investigaciones, los docentes deben fortalecer sus conocimientos en aplicaciones tecnológicas; así mismo, el comportamiento de la actual generación en materia de elección de una carrera universitaria está relacionado con el interés primordial de lograr una vinculación laboral.

\section{AISLAMIENTO SOCIAL POR COVID-19 EN LA AFECTACIÓN DE LA SALUD MENTAL: CASO UNIVERSIDAD DE LA GUAJIRA. Artículo} de investigación a cargo de las académicas Clara Judith Brito Carrillo, Elvis Eliana Pinto Aragón, Delvis Muñoz Rojas, Universidad de La Guajira. Analiza los estragos del aislamiento social por COVID-19 en la salud mental de los estudiantes de la Universidad de La Guajira, Colombia. A manera de conclusión se denota una multiplicidad de distintas consecuencias en el ámbito educativo, dado que los estudiantes manifestaron una desmotivación para seguir estudiando, debido a la falta de herramientas y habilidades tecnológicas tanto por parte de ellos como de los docentes. La calidad educativa, así como el rendimiento escolar, disminuyó en las modalidades implementadas por las instituciones, dejando a los alumnos con una carga de trabajo mayor al conocimiento adquirido durante la impartición de materias; surgió en parte de la comunidad estudiantil la idea de abandonar los estudios a causa de las limitantes ya mencionadas.

ESTUDIO SOBRE EL MODELADO DEL CHORRO DE INYECCIÓN EN MOTORES DE COMBUSTIÓN INTERNA DIESEL CON APLICACIONES DIDÁCTICAS. Artículo de investigación obra de los académicos Mawency Vergel, Jhan Piero Rojas, 'Sofia Orjuela, Universidad Francisco de Paula Santander. Aborda los métodos y diseños experimentales didácticos - investigativos usados para modelar el chorro líquido en motores de combustión interna Diésel y la validación de dichos modelos; además, busca conocer la 
tendencia actual en este campo. También, conocer los principios físicos y la tendencia tecnológica del proceso de inyección, con el fin de cimentar una base para futuros trabajos sobre el tema. Este acercamiento encontró que la tendencia actual es la de mejorar la combustión para producir menor materia contaminante, para así poder cumplir con las normativas vigentes, además se demostró que el modelado CFD es la herramienta más usada por los investigadores en el último año, debido a su relativo bajo costo y su precisión, en términos tecnológicos la inyección directa sigue siendo la opción más investigada y la cavitación presenta ahora un campo relativamente virgen de investigación debido a su complejidad, estas razones hacen a la cavitación un campo interesante de investigación, por otra parte el uso de bancos de inyección es la forma de validación más usada, debido a la facilidad para observar el proceso y controlar los distintos parámetros que gobiernan a la inyección, por otro lado los métodos ópticos son una opción interesantes para recoger datos debido a su exactitud.

\section{ESTUDIO SOBRE LAS CARACTERÍSTICAS} DE VIDA DE LAS FAMILIAS DE PERSONAS CON DISCAPACIDAD EN MEDELLíN. Artículo de investigación obra de los académicos Juan Paulo Marín Castaño, Nicolás Antonio Sepúlveda Tamayo, Enoc Valentín González Palacio, Politécnico Colombiano Jaime Isaza Cadavid- Universidad de Antioquia. Sobre las características de vida de las familias cuidadoras de personas con discapacidad en la ciudad. En los resultados hallados se puede constatar que la posición de ser cuidador de una persona con discapacidad, transforma las condiciones de vida, dado que aspectos vinculados con la economía, la educación, la salud y el acceso a servicios públicos se ven perjudicados. A través del estudio se puede concluir que es indispensable que se promuevan modificaciones en las entidades gubernamentales y en la política pública, de modo que se produzcan mayores oportunidades, tanto para las familias como para las personas con discapacidad.

\section{ESTIMACIÓN DE PARÁMETROS DE UN} MODELO MATEMÁTICOENLATRANSMISIÓN DE VIH/SIDA EN PASTO-COLOMBIA. Artículo de investigación elaborado por Marilin Nathalya Guerrero Laos, Miller Cerón Gómez, Eduardo Ibargüen Mondragón, Universidad de Nariño. Desde 1981, el VIH arrasó con una gran cantidad de vidas, incluidos niños, adolescentes y adultos. Propuesta de un modelo matemático en ecuaciones diferenciales ordinarias para estudiar la dinámica de transmisión de $\mathrm{VIH}$ en la Ciudad de Pasto-Colombia. A pesar de que la muerte causada por VIH se ha reducido gracias a medicamentos "antirretrovirales", este se sigue propagando en la población. El modelo considera la población segmentada en tres compartimentos: susceptibles, infectados y protegidos. Para el análisis del modelo matemático se usaron datos de personas infectadas por VIH durante el año 2008 hasta el 2018, los cuales sirvieron como base para la estimación de parámetros.

\section{CARACTERIZACIÓN SOCIOECONÓMICA DE LA POBLACIÓN MIGRANTE EN EL MUNICIPIO DE VILLA DEL ROSARIO - NORTE DE} SANTANDER. Artículo de investigación autoría de los académicos César Augusto Hernández Suárez, Raúl Prada Núñez, William Rodrigo Avendaño Castro, Universidad Francisco de Paula Santander. En torno al éxodo de los venezolanos a Colombia y particularmente al Municipio del Villa del Rosario - Norte de Santander. Los resultados demuestran que los migrantes encuestados no tienen una vivienda digna donde habitar y carecen de los servicios públicos necesarios para tener una calidad de vida aceptable, a demás no tienen la alternativa 
de acceder a los servicios de salud básicos para garantizar su sobrevivencia en caso de enfermedad grave.

PROCESOS DE MODELACIÓN MATEMÁTICA Y MODELACIÓN ESTADÍSTICA EN TIEMPOS CONTEMPORÁNEOS: SIMILITUDES Y DIFERENCIAS. Artículo de investigación elaborado por la académica colombiana, Rosa Virginia Hernández, Universidad Francisco de Paula Santander. Pone de presente similitudes y diferencias entre Modelación Matemática y Modelación Estadística en tiempos contemporáneos, con el propósito de proporcionar criterios en la selección de contenidos de referencia e implementar escenarios de aprendizaje apropiados e, incluso, diseñar secuencia de actividades permanentes que le permitan al estudiante adquirir la capacidad de construir el conocimiento científico. Para ello, se lleva a cabo un estudio documental de carácter crítico-interpretativo que permitió avanzar en el estado del arte en el que se destaca la gran evidencia de teorías y aportes investigativos en todos los niveles educativos hacia la Modelación Matemática y la Modelación Estadística. Se concluye sobre la diversidad de concepciones entre la Modelación Matemática y la Modelación Estadística, partiendo de los significados de palabras como modelo, modelado, matematización, modelaje, modelización y modelación.

\section{STUDY ON THE ENERGY OVERVIEW OF} THE INDUSTRIAL BEVERAGE SECTOR IN COLOMBIA. Artículo de investigación elaborado or los académicos Jhan Piero Rojas, Mawency Vergel, Sofia Orjuela, Colombia. In the Latin American region, the issues of efficiency in energy consumption and clean energy sources become more important every day, and worldwide, these issues are of great importance because a progressive change in the use of energy is required for reasons such as climate change, polluting emissions and forecasting of a shortage of fossil or non-renewable fuel reserves. The Colombian government is no stranger to these issues, and since 2001, energy efficiency plans have been implemented, creating a nationwide program for the proper use of energy sources that seeks, through analysis of the national energy landscape, the implementation of technical and technological actions that improve energy efficiency in all sectors of the country. This paper shows the study made to the industry of (number of companies) in the beverage sector, which characterized the use of different types of energy such as natural gas and electric energy and the transformation of these in their processes of production, which seeks to determine how efficiently the potential of energy resources is used. Only in the energy consumption for lighting were savings potentials of up to $50 \%$ detected. It is also shown how, through legislation and studies conducted under the direction of the Energy Mining Planning Unit, the aim is to have a more competitive industrial sector against international markets, specifically taking the industrial sector of beverage production as a sample.

\section{LA COMPRENSIÓN DE LOS POLÍGONOS POR MEDIO DEL GEOGEBRA EN ESTUDIANTES DE GRADO SÉPTIMO. Artículo de investigación} elaborado por los académicos Deisy Tatiana Cuervo Lancheros, Carlos Andrés Fonseca Cuervo, Omaida Sepúlveda Delgado, Universidad Pedagógica y Tecnológica de Colombia. Presenta el desarrollo de un trabajo de investigación que tiene entre sus objetivos el reconocimiento de conceptos básicos de geometría por medio del diseño y aplicación de una prueba diagnóstica que involucra la utilización del software dinámico GeoGebra. Entre los resultados evidenciados a partir de la implementación de esta prueba diagnóstica, se identificaron algunas dificultades especialmente, en el desarrollo en forma autónoma y completa 
de situaciones problema y el trabajo en grupo, relacionado con el intercambio y la comparación de la información con los compañeros. Por tanto, luego de los análisis a las situaciones planteadas, se concluye que es importante implementar en el diseño de estas secuencias didácticas, situaciones adidácticas, ya que estas permiten la construcción autónoma del saber matemático, y fortalecen el aprendizaje geométrico y el trabajo en grupo.

VIOLENCIA INTRAFAMILIAR EN TIEMPO DE PANDEMIA EN UNA COMUNA DEL DISTRITO ESPECIAL TURÍSTICO Y CULTURAL EN EL DEPARTAMENTO DE LA GUAJIRA. Artículo de investigación obra de las académicas Clara Judith Brito Carrillo, Juannys Chiquillo Rodelo, Yenifeth Omaira Blanco Torres, Universidad de La Guajira. Analiza el aumento de la violencia intrafamiliar en tiempos de pandemia en el departamento de La Guajira, municipio de Riohacha. Los resultados muestran en los victimarios inconvenientes de tipo psicológico y social, carencias afectivas, inadecuadas relaciones parento- filiares y de convivencia familiar, ausencia de acciones socio psicoeducación en tiempo de confinamiento sobre el fenómeno presentado, y una influencia marcada cultural del papel de la familia en la sociedad. A manera de conclusión, la situación de contingencia y sus diferentes manifestaciones de violencia intrafamiliar requiere medidas por parte de los diferentes entes gubernamentales, además de la implementación de medidas de salud que propendan por mitigar los efectos devastadores sobre las personas en tiempos de confinamiento obligatorio. Aunque las medidas de protección limiten la intervención o haga que sea más restringido desde el área de la salud mental se pueden implementar herramientas desde las instituciones para abordar los efectos nocivos de la violencia intrafamiliar en el departamento de La Guajira, municipio de Riohacha.

\section{ESTUDIO DE LAS CÓNICAS EN ALGUNAS MÉTRICAS: PROPUESTA PARA EL DESARROLLO DEL PENSAMIENTO} ESPACIAL. Artículo de investigación elaborado por los académicos Jesús Adrián Antonio Peña, Cristian Julián Garzón Zipa, Omaida Sepulveda Delgado, UPTC. Sintetiza los resultados más importantes del trabajo investigativo de Antonio y Garzón (2017) referente al estudio geométrico y analítico de las cónicas cuando se abordan desde otras métricas (taxi, máximo y discreta) y reflexionar acerca de la forma en que se está enseñando esta temática, dado que desde el Ministerio de Educación Nacional hay una preocupación por el detrimento del pensamiento espacial y hay una necesidad de rescatar los análisis de tipo intuitivo y crítico en los estudiantes. En muchas ocasiones se asocia una cónica con una representación gráfica determinada, en esta investigación se lograron nuevas representaciones tanto algebraicas como geométricas de las cónicas cuando se cambia la forma de medir, con esto entendemos mejor la definición de cada cónica como lugar geométrico, dejando claro que hay varios caminos para abordar las cónicas en un proceso de enseñanza escolar que favorecen el desarrollo del pensamiento espacial.

PERCEPCIÓN DE JÓVENES ESCOLARIZADOS SOBRE EL ÉXODO DE MIGRANTES VENEZOLANOS: ANÁLISIS DE LAS ACTITUDES XENOFÓBICAS EN CONTEXTOS FRONTERIZOS DESDE LO SUTIL Y LO MANIFIESTO. Artículo de investigación de los académicos Audin Alioso Gamboa Suárez, César Augusto Hernández Suárez, William Rodrigo Avendaño Castro, UFPS. Muestra las percepciones que tienen jóvenes escolarizados sobre la migración desde el prejuicio sutil y 
manifiesto a la población venezolana. Los resultados muestran que se evidencia un rechazo radical para entablar relaciones cercanas hacia la población migrante en el contexto social, familiar y laboral. fFenómeno que durante los últimos años ha generado preocupación entre investigadores en el campo de las Ciencias Sociales y Humanas.

\section{DETERMINACIÓN DEL ERROR INDUCIDO} AL USAR UNA RELACIÓN ESFUERZODEFORMACIÓN LINEAL EN EL CALCULO DEL MOMENTO DE FLUENCIA DE UNA SECCIÓN DE VIGA DE CONCRETO REFORZADO. Artículo de investigación obra de los académicos Jorge Fernando Márquez Peñaranda, Mawency Vergel Ortega, José Leonardo Jácome Carrascal, UFPS. En torno al cmportamiento de una sección de concreto reforzado de acuerdo con el modelo simplificado y contrasta sus resultados con lo que se obtiene al usar un modelo exacto basado en la función propuesta por Hognestad en 1955 que aún es pieza central de la literatura relacionada. Como conclusión se observa de manera interesante que los resultados predichos por el modelo simplificado se ajustan bien a los obtenidos usando el mayor rigor matemático.

\section{REFERENCIAS BIBLIOGRÁFICAS}

Cañaveral Londoño, D,C.,

Rincón Angarita, D.M.,

López Jiménez, L. M.-P., \& Rengifo-Castañeda, C.-A. (2018).

La comprensión de la Justicia desde una perspectiva interdisciplinar a partir de la Idea de Justicia de Amartya Sen. Armenia:

Departamento de comunicaciones y mercadeo. Universidad La Gran Colombia
ESTRATEGIA DE INTERVENCIÓN EN COMUNIDADES PARA LA CONSERVACIÓN DEL MEDIO AMBIENTE: UNA APROXIMACIÓN METODOLÓGICA PARA LA INVESTIGACIÓN SOCIAL. Artículo de investigación de los académicos.Erwin Hernando Jácome Castilla, Naydú Judith Jácome Castilla, Eduardo Luis Jácome Castilla, Universidad Francisco de Paula Santander. Enseña resultados de aporte metodológico desarrollados en 12 investigaciones, las cuales tuvieron como actores clave instituciones educativas del Norte de Santander y adultos mayores asentados en la cuenca del Río Pamplonita. Se construyeron lazos fuertes con comunidades locales que no habían abordado la temática ambiental desde su contexto, problemáticas y necesidades. Se construyó una guía metodológica que caracteriza el proceso de sensibilización. Como resultado de esto, se diseñó el método que permite mediante estrategias, fortalecer la relación ser humano/medioambiente para generar cambios socio ambientales donde se conserve y proteja los recursos naturales. 